\title{
Experiencias de cuidado de las mujeres boyacenses, sometidas a mastectomía por cáncer de mama
}

Experiences of caring for Boyaca women submitted to mastectomy by cancer de seno

Experiências do cuidado às mulheres Boyaca apresentadas à mastectomia pelo cancer de seno

\author{
Marianne Arlette Durán Avendañoํㅜ, Carmen Helena Ruiz de
}

Cárdenas $^{2}$

\begin{abstract}
${ }^{1}$ Magister en enfermería, Universidad de la Sabana; Especialista en Salud Ocupacional y Protección de riesgos laborales Universidad Pedagógica y Tecnológica de Colombia; Enfermera, Universidad Pedagógica y

Tecnológica de Colombia; Docente Escuela de Enfermería, Universidad Pedagógica y Tecnológica de Colombia. Correo electrónico: marianne.duran@uptc.edu.co

${ }^{2}$ Magister en Enfermería énfasis cuidado materno perinatal, Universidad Nacional de Colombia; Especialista en Enfermería perinatal, Universidad Nacional de Colombia; Enfermera, Colegio Mayor de Nuestra Señora del Rosario; Docente Maestría en Enfermería, Universidad de la Sabana. Correo electrónico: ccruizd@unal.edu.co

Cómo citar este artículo en edición digital: Durán Avendaño M.A. E Ruiz de Cárdenas C.H. (2019). Experiencias de cuidado de las mujeres boyacenses, sometidas a mastectomía por cáncer de mama. Cultura de los Cuidados (Edición digital), 23(53). Recuperado de http://dx.doi.org/10.14198/cuid.2019.53.13

Correspondencia: Carrera 3. ${ }^{a}$, numero 3. ${ }^{-}-10$ barrio Coservicios Tunja Boyacá, Colombia Correo electrónico de contacto: marianne.duran@uptc.edu.co Recibido: 16/11/2018; Aceptado: 12/01/2019
\end{abstract}

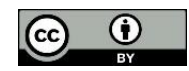

\section{ABSTRACT}

Objective: To describe the core experiences of Boyaca women, undergoing mastectomy for breast cancer, during 2017.

Methodology: Qualitative ethnographic type according to the small-scale ethnonursing, of Leininger's transcultural theory. The participants included ten women key informants and eight general informants. The analysis of the information obtained in the interviews was developed in four phases: 1. Collection, description and documentation of raw data; 2 . Identification and categorization of the descriptors and components; 3. Contextual and pattern analysis; 4. Central themes, research findings, theoretical formulations, and recommendations. The analysis was made taking into account the guide proposed by Leininger. The factors of the social and cultural structure, of the model of the rising Sun The experiences of care experienced by women were described identifying a total of two hundred and eight codes and twenty recurring patterns, which were analyzed and according to the level of coding were labeled and coded giving rise to six themes: 1. The accompaniment an important part in the care of the woman subjected to Mastectomy; 2. The strength of faith; 3. 
Unpleasant effects of treatment; 4 . Experience lived; 5. Importance of care in recovery; 6. Feeding basic support in recovery.

Key Words: Mastectomy, cultural care, breast cancer.

\section{RESUMO}

Objetivo: Descrever as experiências de cuidados que têm as mulheres Boyacenses submetidos a mastectomia por câncer de mama durante 2017.

Metodologia: Qualitativa etnográfica de acordo com etnoenfermagem pequeno intervalo, a teoria transcultural de Leininger. Os participantes incluíram 10 informantes-chave e 8 informantes gerais. A análise das informações obtidas nas entrevistas foi desenvolvida em 4 fases: 1 . Coleta, descrição e documentação de dados brutos; 2. Identificação e categorização dos descritores e componentes; 3. Análise contextual e padrão. Temas centrais, resultados de pesquisas, formulações teóricas e recomendações. A análise foi feita levando em consideração o guia proposto por Leininger. Os fatores da estrutura social e cultural, do modelo do Sol nascente. Cuidar experiências vividas por mulheres que identificam total de 208 códigos e 20 padrões recorrentes que foram analisados e codificação nível rotulados e codificados dando origem a seis questões: 1 . Acompanhando uma parte importante no cuidado descrito mulher submetida a mastectomia; 2. A força da fé; 3. Efeitos desagradáveis do tratamento; 4 . Experiência vivida; 5. Importância do atendimento na recuperação; 6. Alimentar suporte básico na recuperação.

Palavras chave: Mastectomia, cuidado cultural, câncer de mama.

\section{RESUMEN}

Objetivo: Describir las experiencias de cuidado que tienen las mujeres Boyacenses, Sometidas a mastectomía por cáncer de mama, durante el año 2017.

Metodología: Cualitativo de tipo etnográfico según la Etnoenfermería de pequeño alcance, de la teoría transcultural de Leininger. Participaron 10 mujeres informantes claves y 8 personas informantes generales. El análisis de la información obtenida en las entrevistas, se desarrolló en 4 fases: 1. Recolección, descripción y documentación de datos en bruto; 2 . Identificación y categorización de los descriptores y componentes; 3. Análisis contextual y de patrones; 4 . Temas centrales, hallazgos de investigación, formulaciones teóricas, y recomendaciones. El análisis se realizó teniendo en cuenta la guía propuesta por Leininger. Los factores de la estructura social y cultural, del modelo del Sol naciente. Se describieron las experiencias de cuidado vividas por las mujeres identificando en total 208 códigos y 20 patrones recurrentes, que se analizaron y según el nivel de codificación se etiquetaron y codificaron dando origen a seis temas: 1 . El acompañamiento una parte importante en el cuidado de la mujer sometida a Mastectomía; 2. La fuerza de la fe; 3. Efectos desagradables del tratamiento; 4. La experiencia vivida; 5. Importancia del cuidado en la recuperación; 6 . Alimentación soporte básico en la recuperación.

Palabras clave: Mastectomía, cuidados culturales, cáncer de seno.

\section{INTRODUCCIÓN}

El cáncer de mama está asociado a diversos factores genéticos, medioambientales $\mathrm{y}$ conductas humanas, que van desde la dieta, 
la reducción de ejercicios, la obesidad, la exposición al humo del tabaco, hasta la postergación de la maternidad y la disminución de la lactancia. También se atribuye el cáncer de mama a las emociones reprimidas, la depresión, el aporte nutricional deficiente, y el excesivo consumo de cafeína. Estas creencias son frecuentes, erróneas y tienden a provocar sentimientos de culpabilidad (Mejía 2011). La mujer influenciada por su entorno construye significados sobre el cáncer, sus secuelas frente a su imagen y al afrontamiento de cambios, lo cual es decisivo en el manejo y desarrollo de tratamientos. En ese sentido, en el proceso de adaptación influye el escenario sociocultural representado por dos condiciones: el apoyo social y los imaginarios simbólicos sobre el significado del cáncer, las cuales facilitan $\mathrm{u}$ obstaculizan el proceso de adaptación. (Carvalho-Fernández 2005). “Las mamas de la mujer son consideradas un símbolo esencial de la feminidad desde tiempos remotos". La mastectomía incluye trastornos como, depresión, sensación de falta de feminidad, una imagen corporal negativa y la pérdida del interés sexual. La mujer que se somete a reconstrucción mamaria, suele obtener una sensación de apariencia física mejorada, con aumento de la confianza en sí misma y de la autoestima. (Mejía 2012). La cultura influye en el modo como la mujer vivencia el cáncer, especialmente con la mastectomía, menciona, que, en el modo de ver el mundo, las apreciaciones de orden moral y valorativo, los diferentes comportamientos sociales e incluso las posturas corporales son productos de una herencia cultural, es decir, el resultado de la operación de una determinada cultura (Calvo-Gil en el 2008).

El impacto emocional no sólo depende del pronóstico médico, sino que se basa en las creencias previas que tenga la paciente respecto al cáncer de mama, estas creencias provocan sentimientos de culpabilidad en la mujer. Así mismo existen variables individuales que influyen en la duración de dicho impacto, tales como: la edad, importancia en la apariencia física y las creencias sobre los tratamientos; dependiendo del tipo de intervención dependerá el tipo de respuesta emocional que experimenta la paciente. (Mejía, 2012). Las mujeres que son diagnosticadas con cáncer de seno requieren de cuidados de enfermería donde se den espacios para reconocer creencia, valores espirituales, expresar emociones de tal forma que las emociones positivas en ellas mejoren y aumenten su periodo de vida, disminuyan el estrés, mejoren su respuesta inmunológica y predisposición a tener hábitos de vida sanos (Rodríguez Y., en el 2013). Dentro del contexto del Departamento de Boyacá, son de importancia sus características sociodemográficas, geográficas y climáticas influyentes en las costumbres de sus poblaciones, al ser uno de los departamentos en Colombia con mayor número de municipios (123 agrupados en 11 provincias) y al estar en el centro del país, recibe variedad de influencia cultural por los departamentos aledaños; es así que hacia el norte se mezcla la cultura de los Santanderes, hacia occidente con la cultura llanera de Colombia y de Venezuela y más arriba hacia al nor-occidente con la cultura indígena U'wa y también al sur oriente con Cundinamarca y parte de Antioquia.

En todo el departamento se encuentran elementos culturales que mezclan las tradiciones de los pueblos aborígenes que habitaron la región con las costumbres españolas, impuestas durante la colonia (Seminario conciliar de Tunja, 2012). Las creencias sobre la medicina popular y la herbología son la base fundamental para las 
curaciones. Los campesinos boyacenses creen con fervor en los yerbateros, curanderos y mediquillos, la medicina popular tradicional presenta una gran difusión en Boyacá; se relaciona con el curanderismo y el yerbaterismo que tienen hondas raigambres indígenas y españolas. (Araque, 2013). En el campo religioso también se mantiene la tradición oral a través de las costumbres y expresiones lingüísticas particulares de los yerbateros, los hechiceros o brujos, que aún hoy, subsisten en las diferentes provincias del departamento de Boyacá. (Araque, 2013). Con respecto al manejo de otras enfermedades y específicamente al manejo del cáncer de mama en nuestra región no se ha logrado encontrar suficiente información, esto fundamenta la necesidad de conocer desde un abordaje cultural las experiencias de cuidado en las mujeres que han sido sometidas a una mastectomía por cáncer de seno.

Las principales consecuencias de no tener presente los enfoques culturales en los sistemas de asistencia de Enfermería son: Pacientes que reciben un cuidado por parte de Enfermería el cual no está relacionado con sus creencias, valores y estilos de vida, creando conflictos culturales, insatisfacción, tensiones, así como preocupaciones éticas o morales; Cuando se realiza un diagnóstico de Enfermería sin tener en cuenta los factores culturales de los pacientes, se puede obtener un resultado desfavorable, en muchas ocasiones con consecuencias graves frente a las metas propuestas. El conocimiento cultural para Enfermería implica proveer un cuidado cultural, en el cual el profesional debe poseer habilidades que faciliten la competencia cultural en el mismo, lo que significa aceptación y respeto por las diferencias culturales; sensibilidad para entender como esas diferencias influyen en las relaciones enfermero- paciente $\mathrm{y}$ viceversa, y habilidad para buscar estrategias que mejoren los encuentros culturales de acuerdo a las necesidades manifestadas por el paciente (Ibarra Mendoza y Siles González, 2006). Objetivo: Describir las experiencias de cuidado que tienen las mujeres Boyacenses, Sometidas a mastectomía por cáncer de seno, durante el año 2017.

\section{MARCO CONCEPTUAL Y METODOLÓGICO}

Para el marco conceptual se tuvo en cuenta los conceptos emergentes desde el fenómeno, estableciendo los conceptos de; experiencias de cuidado influenciadas por la cultura y mujer sometida a mastectomía por cáncer de seno; así como establecidos en la teoría de los cuidados culturales de Madeleine Leininger como son cuidado genérico o émico, cuidado profesional o ético y los tres modos de acción (Leininger, 2006).

Metodológicamente esta investigación se realizó con un enfoque cualitativo de tipo etnográfico el cual aplica los elementos básicos de la Etnoenfermería de pequeño alcance, de la teoría transcultural de Leininger.

Tipo de muestreo: La recolección de la información fue progresiva y estuvo sujeta a resultados de los hallazgos, por lo cual propone 5 elementos: pertinencia, adecuación, conveniencia, oportunidad y disponibilidad. (Sandoval, 1996)

Técnicas de recolección de la información: Se utilizó la entrevista a profundidad de Spradley, estableciendo preguntas descriptivas, estructurales y de contraste, también, el diario de campo como herramienta de corroboración y contraste, y entre otros, los facilitadores establecidos por Leininger como son Extraño amigo, OPR (Observación, Participación, Reflexión) y el 
Modelo del sol Naciente.

Procedimiento: Se llevó a cabo por medio de la inmersión al campo, posteriormente las Entrevistas y por último la transcripción de la información.

\section{RESULTADOS Y DISCUSIÓN}

Características de los participantes: Fueron 10 mujeres sometidas a mastectomía como informantes clave y 7 informantes generales que aportaban informacional adicional o corroboraban la recibida pos las informantes clave. La edad de las informantes clave oscilaba entre los 35 y más de 60 años; todas las mujeres residen en el departamento de Boyacá desde hace más de 5 años, la procedencia de las mujeres correspondía a 8 mujeres del departamento de Boyacá de su área rural y urbana de las diferentes provincias del departamento, y 2 mujeres procedentes de Santander y Cundinamarca; al finalizar la investigación 2 mujeres fallecieron por complicaciones del cáncer. El promedio de entrevistas fue de 1 a $4 \mathrm{y}$ los informantes generales fueron principalmente los hijos, la madre y el esposo.

Análisis de datos: El análisis se dio según las fases establecidas por Leininger el cual se describe en la siguiente tabla:

\section{TABLA 1: Análisis por fases según Leininger}

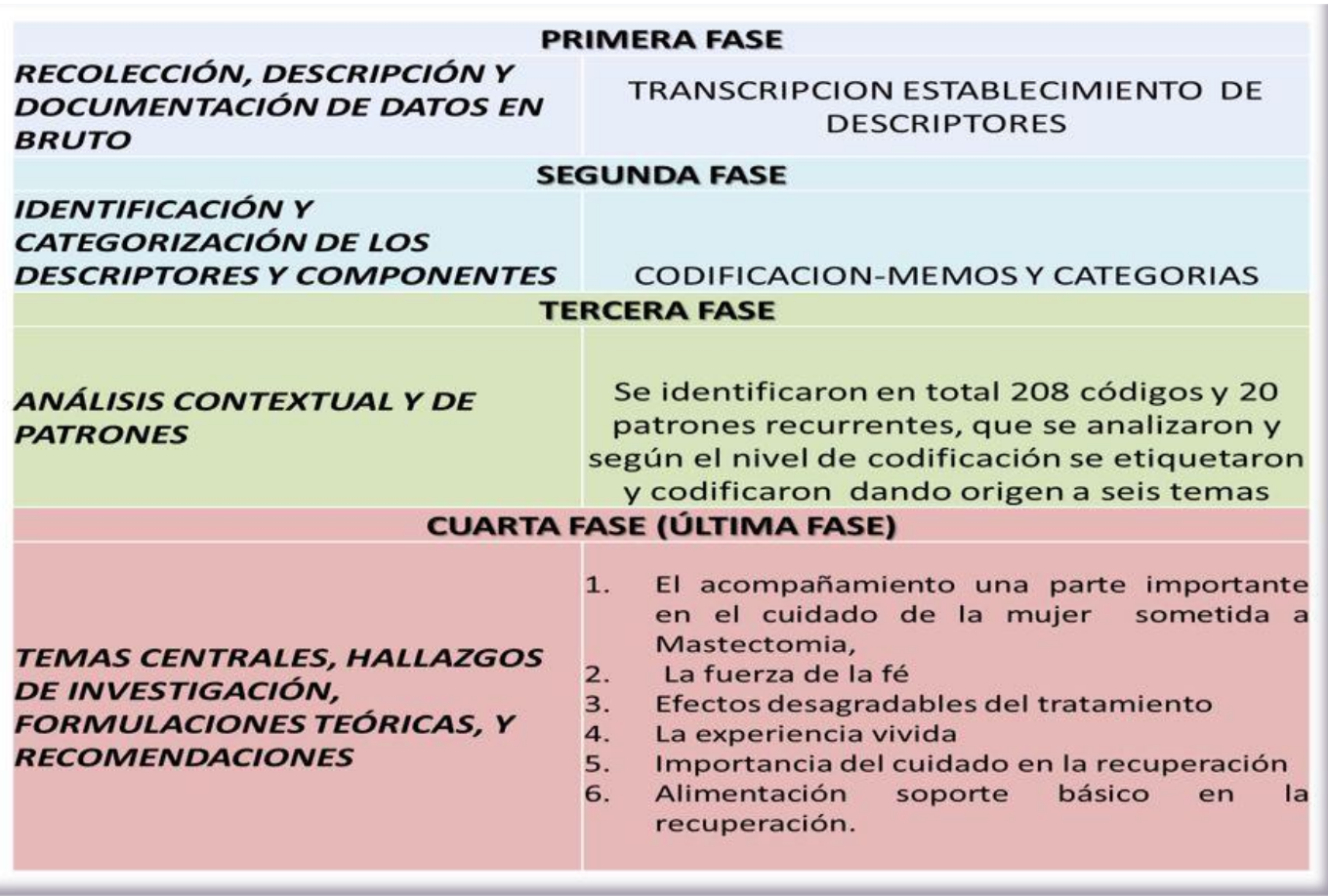




\section{DESCRIPCIÓN DE LOS TEMAS}

\section{TEMA 1. EL ACOMPAÑAMIENTO UNA PARTE IMPORTANTE EN EL CUIDADO DE LA MUJER SOMETIDA} A MASTECTOMIA

PATRON 1. ACOMPAÑAMIENTO FAMILIAR

PATRON 2. ACOMPAÑAMIENTO

\section{PATRON 3. ACOMPAÑAMIENTO DE ENFERMERIA}

El acompañamiento una parte importante en el cuidado de la mujer sometida a mastectomía, permitió observar la relación estrecha entre el acompañamiento de la familia como parte vital del proceso para la mujer, así como el acompañamiento profesional experimentando por las mujeres de forma positiva y también con algunas observaciones para mejora.

“... Pero entonces mi familia. Pues el deseo de que me recuperara insistían y jodían... en el proceso mi familia fue muy importante pues todos me ayudaban en lo que podían. Me dieron moral sabe que sobre todo con mis nietos..." EC5P4Pf4.

"Ellas si te canalizaban y ponían las quimios pero pues contacto no mucho, ellas hacían lo que les tocaba y ya" EC3P5Pf3.

"la enfermera no mucho, más que todo con los doctores y pues si de las niñas que nos colaboran si para los procedimientos y eso... Pero pues no veo mucho el de enfermería" EC2P4Pf1.

TEMA 2. LA FUERZA DE LA FÉ

\section{PATRON 1.FÉ EN} DIOS
PATRON 2. FÉ

EN LA VIRGEN
PATRON 3. LA ORACION Y EL USO DEL AGUA BENDITA
PATRON 4.FE EN MÉDICOS BRUJOS
La fuerza de la fe, corroboró el fuerte arraigo en la cultura boyacense del factor religioso y su influencia en la salud de las mujeres y sus familias, y como la vivencia del cáncer aumenta de forma significativa el acercamiento a la religión y la Fe en Dios, uso de la oración y fe en médicos brujos y misas de sanación.

"eso yo digo todo gracias a Dios que me permite hacer cosas por mí misma, que me dio fuerzas y no me dejo deprimirme" EC2P3Pf3. "encomendarse a papito Dios, el sana si tiene uno fe" EC2P8Pf4.

"también voy a mis misas de sanación en Soraca... al divino niño en Tunja y al Topo y pues la $m$ isa del domingo eso es sagrado mi Diosito está conmigo siempre" EC9P4Pf1.

"la verdad he tratado con todo lo que se también...y en el terminal de Tunja hay un médico brujo muy bueno... el llanero esteban le dicen, me da unas bebidas muy buenas y me hace unos rezos..." EC9P4Pf1. 
PATRON 1. EFECTOS DE LA

QUIMIOTERAPIA
PATRON 2. EFECTOS DE LA RADIOTERAPIA
Los efectos desagradables son más fuertes en la quimioterapia presentando alteraciones de orden físico y psicológico. La radioterapia considerada como menos agresiva, con la mastectomía se encontró incomodidad sin alteraciones físicas, con gran impacto con respecto a su imagen personal y efectos en su autoestima.

"a mí el cabello sí, no se me quería caer, entonces eso fue lo que me dolió si, el médico me dijo se lo quita porque se tiene que caer" EC1P6Pf.

Los vómitos uno de los efectos más desagradables:

"los vómitos a veces me daban a veces no, así sea frecuente uno a eso si no se acostumbra" EC2P4Pf2.
Otros efectos menos comunes fueron expresados:

"ya iba yo por la mitad de las quimios, se me cayeron un día dos dientes" EC1P6Pf1.

"Por la medicina mis uñas eran negras, Empecé a notar la medicina por las uñas de manos y pies. Eso fue lo que me dio rarísimo". EC1P6Pf6.

Con respecto a la radioterapia las mujeres pueden presentar diversos síntomas entre ellos cansancio y alteraciones dermatológicas:

"porque con las radios la piel se le pone a uno horrible tiesa y rasposa y la cremita sirve mucho" EC2P8Pf3.

\section{TEMA 4. LA EXPERIENCIA VIVIDA}

PATRON 1. DENTIFICANDO POSIBLES CAUSAS

PATRON 4. DECISION DEL TRATAMIENTO
PATRON 2. SINTOMAS INICIALES

PATRON 3

DIAGNOSTICO

PATRON 5. IMAGEN CORPORAL
PATRON 6 COMPARTIR CON OTROS
La experiencia vivida, permite ver como las participantes identifican las posibles causas, siendo el factor genético el más predominante, las mujeres tomaron la decisión de realizarse la mastectomía bilateral total como prevención de futuras reapariciones, Frente a la imagen personal se observó como para las mujeres la mastectomía generó sentimientos de mutilación, estar feas e incompletas $y$ sentirse poco mujeres, pero todas coincidían en la importancia de la pérdida con respecto 
al mejoramiento de su salud. El compartir con otros esta experiencia generó en las mujeres, motivación de ayuda y colaboración para prevenir en las personas sentimientos negativos experimentados por ellas como la incertidumbre, el dolor y la falta de información.

"A mis dos tías maternas y unas primas les encontraron el gen, es decir el gen viene por la parte materna..." EC3P1Pf3.

Cada una de las participantes expreso la forma como identificaron el cáncer:

"Miro la blusa y verde, me mire y todo verde... verde y los dos senos" E1P12Pf6.

"Mire uno de mujer los senos son importantes pero créame que yo creo que a todos con pasa bueno ya a mí no, ver los senos como belleza únicamente cuando me iban a hacer la cuadrantectomía uno entra en que... virgen del Carmen... lo primero es pensar y ahora seré fea incompleta" EC2P9Pf2.

"bueno yo las ayudo dándoles consejos sobre mi experiencia, como van a sentir, como si nos cuidamos podemos prevenir el cáncer, no solo con mis compañeras de las quimios sino con mi familia y conocidos para que no les llegue a dar cáncer de ningún tipo, cuando no padezcan de nada no les pase nada que uno tenga, entonces que ellas también se cuiden y que estoy muy pendiente a veces de las personas... también que ellas estén bien que no se vayan a enfermar" EC4P7Pf2.

TEMA 5. IMPORTANCIA EN LOS CUIDADOS

EN LA RECUPERACION

\section{CUIDADOS GENERICOS}

PATRON 1. EL

CUIDADO

CULTURAL: USO DE HIERBAS
PATRON 2. CUIDADOS PARA EL

CABELLO

PATRON 3.CUIDADOS CON LA HERIDA

\section{CUIDADOS PROFESIONALES}

PATRON 1.

MANEJO DELA HERIDA
PATRON 2.

CUIDADOS

GENERALES
PATRON 3

INFORMACION

BRINDADA
PATRON 4. PERCEPCION DEL CUIDADO
PATRON 5. CAMBIOS

EN EL ESTILO DE VIDA
Se identificaron cuidados culturales con el uso de hierbas en infusión o en emplastos para el manejo de la herida, para disminuir efectos gastrointestinales, para calmar los nervios y favorecer la digestión, y para evitar la deshidratación.

Se encontró que estos cuidados tradicionales las mujeres los complementaban con el cuidado profesional recibido, siendo los dos cuidados para ellas muy confiables y primordiales para su recuperación.
Dentro del cuidado cultural podemos diferenciar varios códigos entre ellos el uso de hierbas:

"ya de vez en cuando compro el agraz bueno para prevenir anemia... sopas aguas de hierbas y me daban... de hierbas normales manzanilla limonaria y hierba buena para mantener las defensas además que son ricas y siempre tomo...porque no me gusta el tinto entonces sabian que mis agüitas si... si desde antes del cáncer" EC6P3Pf2. 
Las hierbas en infusiones o fueron un factor predominante en las participantes, se encontró, la manzanilla como anti inflamatorio, la caléndula tomada y en emplastos para cicatrizar, diente de león y salvia para limpiar la sangre y para el vómito, agua con canela para la deshidratación, limonaria y hierba buena para mejorar las defensas, marihuana fumada para el dolor en una señora en etapa terminal, jengibre con limón y miel para las defensas, él té verde para la digestión y otros tipos de té para los nervios, otro cuidado referido aunque no es una hierba el uso del agua de arroz para la deshidratación después de los vómitos y el agua de piña como diurético. Todas las mujeres refirieron que la utilización y preparación fue obtenida principalmente por recomendación de las madres o aprendido por ellas o sus abuelas y que la mayoría vienen de tradiciones familiares, y que en muchos casos ellas ya la habían utilizado con anterioridad tanto en enfermedades diferentes al cáncer, en ellas mismas como de sus hijos y familiares.

La información recibida por parte de los profesionales de la salud:

"Pues que me explicaran todo y todo lo que me dijeron hice pero pues que le digan como más sobre las cosas que pueden molestar que le expliquen a uno mejor y bien" EC2P6Pf3.

Frente a la percepción del cuidado la mayoría de las mujeres se sintieron cuidadas, tenidas en cuenta así como para otras se hace necesario un cuidado más individualizado y humano:

-Las experiencias positivas de las mujeres:

"me aplicaban los medicamentos las enfermeras, una era la jefe muy querida y habian auxiliares que nos ayudaban en cositas, todos muy queridos" EC1P8Pf5.

-Y también otras no tan positivas:

"mi caso ahorita por el diagnostico que estoy pasando solamente el papel de la enfermera es el momento de la quimioterapia..." EC3P6Pf1.

\section{TEMA 6. LA ALIMENTACIÓN SOPORTE BÁSICO EN LA RECUPERACIÓN}

La alimentación como soporte básico en la recuperación fue el tema de mayor relevancia en la investigación establecido alimentarse sano y la alimentación saludable.

Encontramos algunos testimonios frente a la alimentación sana:

"pues lo bueno para el cáncer y para evitar que vuelva pues comer son mucha fruta verdura y carne pero de pollo o pescado y evitar galguerías gaseosas y el azúcar refinada..." EC6P3Pf3.

Frente a la alimentación saludable:

“de la alimentación todo era muy nutritivo... pescado pollo... atún pero en agua... jugos de mora, de naranja, de remolacha para fortalecer el sistema inmunológico, la emulsión de Scott también y los micronutrientes..." EC10P3Pf3. 
RELACIÓN CON EL MODELO DEL SOL NACIENTE:

Todos los temas resultantes tienen una interacción especifica con los factores relacionados por Leininger en el modelo del sol naciente, en donde el factor familiar y social fueron predominante en todos los temas, el factor económico, el factor tecnológico con el manejo de información en internet y el factor religioso y filosófico y el factor político solamente fueron evidentes en el tema de acompañamiento, como se muestra en la siguiente gráfica:

\section{GRÁFICA 1: Relación con el modelo del sol naciente}

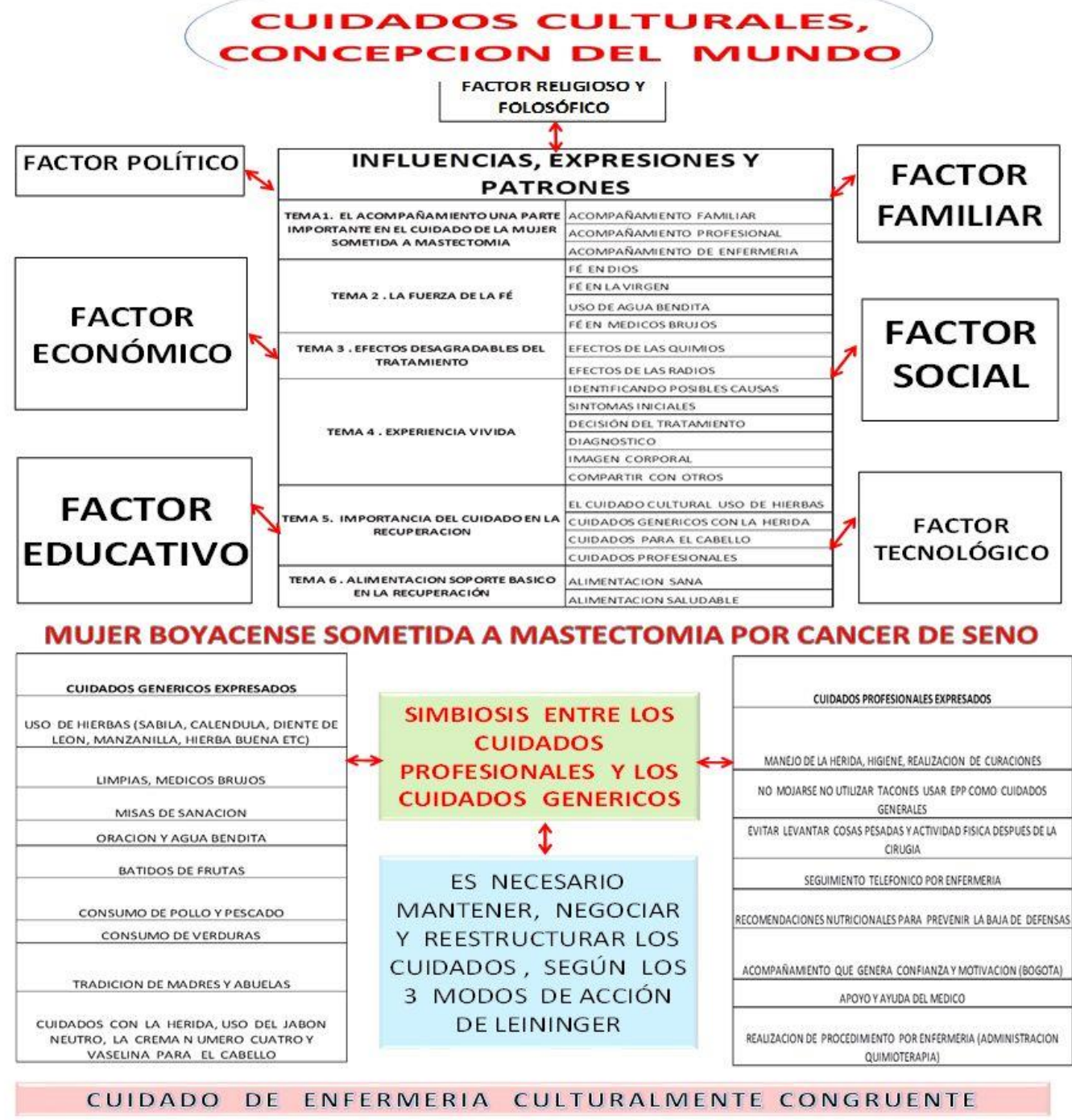




\section{CONCLUSIONES}

- Las experiencias de cuidado de las mujeres boyacenses, sometidas a mastectomía por cáncer de seno, presentan una gran influencia cultural adquirida de generación en generación específicamente de las madres y abuelas, las mujeres participantes mostraron como complementan los cuidados profesionales recibidos con la práctica de cuidados tradicionales percibidos como benéficos para su salud física emocional y espiritual y evidenciado con la literatura.

- Es necesario establecer estrategias de mejoramiento frente al cuidado médico y especializado y más frente al cuidado de Enfermería culturalmente congruente a las mujeres sometidas a mastectomía por cáncer de seno en Boyacá.

- Para las mujeres la experiencia del cáncer y la mastectomía genero cambios importantes en su estilo de vida estableciendo mayor positivismo frente a la vida, cambio radical y muy positivo en la forma de alimentación y les permitió mayor unión y mejoramiento de las relaciones familiares, el único aspecto negativo para ellas fue la disminución de la actividad física por los efectos posteriores a los tratamientos como era la perdida de la fuerza. Se pudo observar la simbiosis que realizan las mujeres entre el cuidado cultural o émic practicado y el cuidado profesional o étic recibido, siendo para ellas en algunos aspectos como el cuidado de la herida el cuidado profesional el predominante y el cultural complementario, mientras que en los demás aspectos era el cuidado cultural el principal y el profesional visto como complementario.

\section{RECOMENDACIONES}

- Continuar indagando sobre el tema propuesto, además realizarla en otras zonas geográficas y otros informantes para poder identificar similitudes $y$ diferencias en el cuidado émic como lo plantea la teoría de Madeleine Leininger.

- Establecer evidencia del beneficio de algunos cuidados culturales además del manejo de las hierbas.

- Para la práctica de la Enfermería en Boyacá se recomienda capacitación inicial sobre el cuidado de pacientes oncológicos a nivel clínico y comunitario, paralelo el reconocimiento de prácticas culturales de cada región.

- Dentro del proceso de formación del Profesional de Enfermería es importante rescatar las tradiciones culturales regionales.

- Continuar enriqueciendo la teoría de Leininger con investigación que evidencie la importancia y la visibilidad de la disciplina en los aportes que pueden orientarse desde los hogares, haciendo acciones directas que deben lograr preservar, mantener o negociar expresiones de cuidado.

- El conocimiento de cuidado profesional y genérico realizado por las mujeres sometidas a mastectomía constituye en una herramienta muy importante para el planteamiento de un Plan de cuidado de Enfermería enfocado en el contexto cultural como aporte al conocimiento disciplinar y ampliando con esto el desarrollo del cuidado culturalmente congruente a la mujer Boyacense sometida a mastectomía por cáncer de seno. 


\section{Limitaciones}

- Las limitación presentada para esta investigación tuvieron que ver más con los tiempos y la disponibilidad de la participantes específicamente debido a la presencia de síntomas generado por los tratamientos.

- Agradecimientos: Agradecemos a las mujeres participantes y sus familiares por permitir conocer sus experiencias, así como a Colombiana de Salud por su colaboración con la Información.

- Financiación: La investigación realizada fue financiada.

\section{BIBLIOGRAFÍA}

- Agüero, L. (2001). Algunos factores que influyen en el distress psicosocial y autoestima en mujeres mastectomizadas. Tesis. Valdivia: Universidad Austral de Chile. Facultad de Medicina.

- Anderson, M.S. \& Johnson, J. (1994). Restoration of body image and self-esteem for women after cancer treatment. Cancer Pract., 5, 345-9. Recuperado de https://www.ncbi.nlm.nih.gov/pubmed/7697072.

- Araque, J. (2013). Tradición oral en la cultura boyacense. Revista Economía y Sociedad. 2(1), 1. Recuperado de http://www.revistasjdc.com/main/index.php/deys o/article/view/266.

- Aungus, J., Miller, K. \& McKeever, P. (2006). Studying delays in breast cancer diagnosis and treatment: critical realism as a new foundation for inquiry. Oncol Nurs Forum E62-70. Recuperado de https://www.ncbi.nlm.nih.gov/pubmed/16858449.

- Blanco, S. (2010). Imagen corporal femenina y sexualidad en mujeres con cáncer de mama. Index Enferm. 19(1), 24-28.

- Calvo, M. \& Narváez, P. (2008). Experiencia de mujeres que reciben diagnóstico de Cáncer de Mamas. Index Enferm, 17(1), 30-33.

- Carvalho, A.F., Mesquita, E., Almeida, I.M. et al. (2005). Aspectos culturales en el proceso de padecer cáncer de mama. Avances en Enfermería, 23, 28-35.

- Ceballos, G. \& Giraldo, C. (2011). Autobarreras de las mujeres al diagnóstico y tratamiento oportuno del cáncer de mama. Aquichan, 11(2), 140-157.

- Daly, H. \& Collins, C. (2007). Barriers to Early Diagnosis of Cancer in Primary Care: A Needs
Assessment of GPs. Irish Med J. Recuperado de http://cancer.ie/pdfs/IMI\%20-

\%20S3952\%20Barriers\%20to\%20Early.pdf.

- Fernández, A. (2004). Alteraciones psicológicas asociadas a los cambios en la apariencia física en pacientes oncológicos. Psico oncología, 1(2), 169-180.

- Giménez, M, A. (1998). Pesquisa do enfrentamiento na practica psico-oncologica, en Carvalho, M.M.M.J, Psico-oncologia no Brasil; resgatando o vivier, Sao Paulo: Summus, 232.

- Giraldo, C., Arango, M.E. (2009). Representaciones sociales frente al autocuidado en la prevención del cáncer de mama. Investigación y Educación en Enfermería, 27(2), 191-200.

- Grana, G. (1998). Ethnic Differences in Mammography Use among Older Women: overcoming the Barriers. Ann Intern Med, 128(9), 773-775.

- Herrera, Z., Conde, L., Correa, M. y Bernal, A. (2014). La competencia cultural como elemento importante de la práctica de cuidado brindado al paciente, por el profesional de enfermería de la Unidad Intermedia de San Javier de la ciudad de Medellín. 1st ed. Medellín: Corporación Universitaria Adventista. Recuperado de http://repository.unac.edu.co:8080/jspui/handle/11 254/151.

- Hewitt, M., Herdman, R. \& Holland, J. (2004). Meeting psychosocial needs of women with breast cancer. National Research Council. Institute of Medicine (US) and National Research Council (US) National Cancer Policy Board.

- Ibarra, T.X. \& Siles, J. (2006). Competencia Cultural: Una forma humanizada de ofrecer Cuidados de Enfermería Cultural. Index de enfermería. Recuperado de https://doaj.org/article/7d1506286dea4374a6422c44 eda4f82b.

- INGE (2015). Estadísticas a propósito del día mundial de la lucha contra el cáncer de mama. México: Instituto Nacional de Geografía y Estadística. Recuperado de http://www.inegi.org.mx/saladeprensa/aproposito /2015/mama0.pdf.

- Instituto Nacional de estadística y geografía. (2015). Estadísticas a propósito del día mundial de la lucha contra el cáncer de mama. México: INEM. Recuperado de http://www.inegi.org.mx/saladeprensa/aproposito /2015/mama0.pdf.

- Leininger, M. (1985). Qualitative research methods in nursing. Orlando: Grune \& Stratton.

- Leininger, M. \& McFarland, M. (2006). Culture care diversity and universality: a Worldwide nursing theory. Sudbury, MA: Jones and Bartlett. 


\section{Cultura de los Cuidados}

- López, Z., González, J., Gómez, M. \& Morales, M. (2008). Impacto psicológico y social de la mastectomía en pacientes operados de cáncer de mama. Rev méd electrón 30(2). Recuperado de URL: http://www.revmatanzas.sld.cu/revista\%20medica /ano\%202008/vol2\%202008/tema01.htm.

- Marriner A. \& Raile, M. Modelos y Teorías de Enfermería. 6. ㄹ ed. Op. cit., pp. 477-479.

- Mejía, M. (2012). Incertidumbre, Calidad de Vida e imagen corporal en mujeres sometidas a Mastectomía. (disertación). Bogotá: Universidad nacional de Colombia. Facultad de Enfermería. Programa de Doctorado en Enfermería.

- Monroy, A. (2002). Cáncer de mama un puente difícil de cruzar. Revista Prometeo; fuego para el propio conocimiento. 31, 39-45. Recuperado de http://www.revistaprometeo.com/prometeo/publi caciones/.

- Pérez, P. \& Florenzano, A. (2016). Mastectomía y sexualidad. Recuperado de http://www.psicosexual.com/2008/03/deseosexual.html.

- Psillakis, J. (1986). Reconstrucción de mamas en pacientes mastectomizadas. En: Coiffman, F., Cirugía plástica, reconstructiva y estética. La Habana: Editorial Científico-Técnica.

- Rivillas, G. \& Ospina, M. (2015). Observatorio Nacional de Cáncer Colombia. Revista Facultad Nacional de Salud Pública. 33.

- Salas, C. \& Grisales, H. (2010). Calidad de vida y factores asociados en mujeres con cáncer de mama en Antioquia, Colombia. Rev Panam Salud Pública. 28(1), 9-18.

- Stein, J., Fox, S. \& Murata, P. (1998). The influence of ethnicity, socioeconomic status, and psychological barriers on use of mammography. $J$ Health Soc Behav, 32 (2), 101-113.

- Suh, E. (2013). Cultural models of female breasts and breast cancer among Korean women. Open Journal of Nursing, 3, 404-413. DOI: 10.4236/ojn.2013.35055. Recuperado de https://www.scirp.org/journal/PaperInformation.a spx?PaperID=36916.

- Terrasse, V. (2016). La batalla mundial contra el cáncer no se ganará únicamente con tratamiento. Who.int. Recuperada de http://www.who.int/mediacentre/news/releases/20 14/cancer-report-20140203/es/. Informe mundial sobre el cáncer 2014, IARC.

- Tobin, M.B., Lacey, H.J., Meyer, L. \& Mortimer, P. (1993). The psicological morbidity of breast cancerrelated arm swelling. Psychological morbidity of lymphoedema. Cáncer. 72, 32-48. Recuperado de https://www.ncbi.nlm.nih.gov/pubmed/8242549 [PubMed].

- Vargas, E. \& Pulido, S. (2012). Significado de las vivencias de las pacientes con diagnóstico de cáncer de mama. Revista ciencia y cuidado. 1, 65-78 Recuperado de https://dialnet.unirioja.es/servlet/articulo?codigo= $\underline{3986765 .}$.

- Vázquez, J., López Tarruella-Cobo, S. \& Martín, M. (2004). Manual de Cuidados Continuos. Sociedad Española de Oncología Médica, Sección de cuidados continuos, 379, 90. Recuperado de http://www.seom.org/seomcms/images/stories/rec ursos/sociosyprofs/documentacion/manuales/cuid Cont/cuidadosContinuos01-20.pdf. 